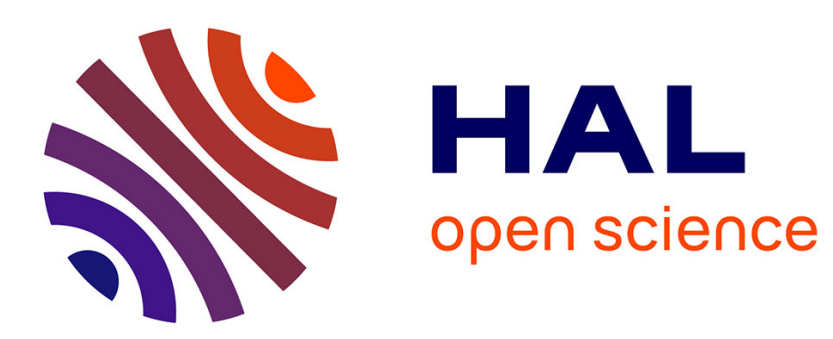

\title{
Kohn anomalies and enhanced superconductivity in simple systems under pressure: Insights from the nearly free electron model
}

\author{
Bruno Rousseau, Ion Errea, Aitor Bergara
}

\section{- To cite this version:}

Bruno Rousseau, Ion Errea, Aitor Bergara. Kohn anomalies and enhanced superconductivity in simple systems under pressure: Insights from the nearly free electron model. Journal of Physics and Chemistry of Solids, 2010, 71 (8), pp.1159. 10.1016/j.jpcs.2010.03.026 hal-00661908

\section{HAL Id: hal-00661908 \\ https://hal.science/hal-00661908}

Submitted on 21 Jan 2012

HAL is a multi-disciplinary open access archive for the deposit and dissemination of scientific research documents, whether they are published or not. The documents may come from teaching and research institutions in France or abroad, or from public or private research centers.
L'archive ouverte pluridisciplinaire HAL, est destinée au dépôt et à la diffusion de documents scientifiques de niveau recherche, publiés ou non, émanant des établissements d'enseignement et de recherche français ou étrangers, des laboratoires publics ou privés. 


\section{Author's Accepted Manuscript}

Kohn anomalies and enhanced superconductivity in simple systems under pressure: Insights from the nearly free electron model

Bruno Rousseau, Ion Errea, Aitor Bergara

PII:

S0022-3697(10)00072-7

DOI: doi:10.1016/j.jpcs.2010.03.026

Reference: PCS 6135

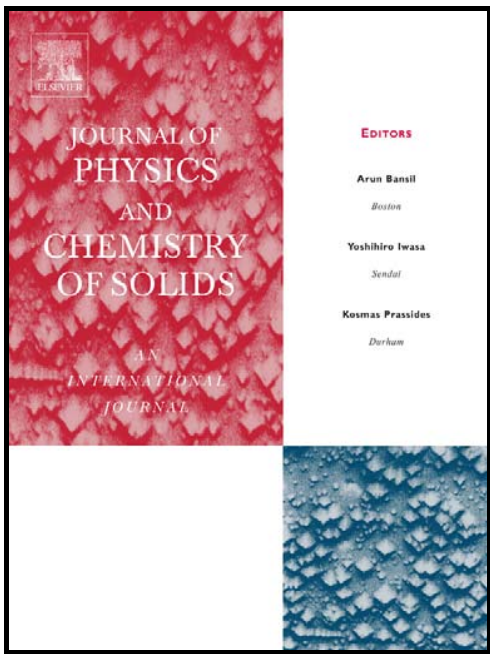

www.elsevier.com/locate/jpcs

To appear in: Journal of Physics and Chemistry of Solids

Cite this article as: Bruno Rousseau, Ion Errea and Aitor Bergara, Kohn anomalies and enhanced superconductivity in simple systems under pressure: Insights from the nearly free electron model, Journal of Physics and Chemistry of Solids, doi:10.1016/j.jpcs.2010.03.026

This is a PDF file of an unedited manuscript that has been accepted for publication. As a service to our customers we are providing this early version of the manuscript. The manuscript will undergo copyediting, typesetting, and review of the resulting galley proof before it is published in its final citable form. Please note that during the production process errors may be discovered which could affect the content, and all legal disclaimers that apply to the journal pertain. 


\title{
Kohn anomalies and enhanced superconductivity in simple systems under pressure: insights from the nearly free electron model
}

\author{
Bruno Rousseau ${ }^{\mathrm{a}}$, Ion Errea ${ }^{\mathrm{a}, \mathrm{b}}$, Aitor Bergara ${ }^{\mathrm{a}, \mathrm{b}, \mathrm{c}}$ \\ ${ }^{a}$ Donostia International Physics Center (DIPC),Paseo de Manuel Lardizabal, 20018, Donostia, Basque Country, Spain \\ ${ }^{b}$ Materia Kondentsatuaren Fisika Saila,Zientzia eta Teknologia Fakultatea, Euskal Herriko Unibertsitatea, 644 Postakutxatila,48080 Bilbo, Basque Country, Spain \\ ${ }^{c}$ Centro Física de Materiales CSIC-UPV/EHU, 1072 Posta kutxatila, E-20080 Donostia, Basque Country, Spain
}

\begin{abstract}
The phonon spectrum of sodium is studied using ab initio methods and a simple nearly free electron model in the pressure range where the fcc phase is stable. It is found that the phonon softening previously reported consist of simple Kohn anomalies, the amplitudes of which are increased under pressure; their relative amplitudes and positions are easily understood in terms of the nearly free electron model. Furthermore, it is shown that superconductivity should be enhanced by pressure because of the increased contribution to the Eliashberg function associated to transverse phonon modes.
\end{abstract}

Key words: phonon softening, pressure, Kohn anomaly, simple metals, superconductivity

\section{Introduction}

Phonon-induced superconductivity is now well understood, and it is fairly straightforward, if technical, to obtain good approximate values of $T_{c}$, the superconducting transition temperature[1]. Calculations are now usually performed using very elaborate, pre-packaged $a b$ initio computation suites. They provide great computational power, but sometimes render the interpretation (indeed, the physics) harder to disentangle from the numerics. In that context, simpler models which still exhibit some of the interesting physics under study become valuable aides to comprehension. With this philosophy in mind, the work presented here provides insight into phonon softening and enhanced superconductivity in simple metals under high pressure using the nearly free electron (NFE) model and local pseudopotentials. These drastic approximations lead to greatly simplified numerics while still allowing the computation of any desired quantity. Although the results cannot be trusted to be quantitatively accurate, the simplified model provides a testing ground for ideas.

It has been noted that the phonons of the alkali metals develop anomalies in the fcc phase which increase in magnitude with pressure[2]. In most of these systems, these anomalies have been linked to the large deformations sustained by their Fermi surfaces, going from the NFE sphere at low density to non-spherical surfaces exhibiting nesting in the pressure range of interest. The wave vectors of the anomalous phonons have been positively correlated to the nesting wave vectors of the Fermi surfaces [2, 3], suggesting that the anomalous phonons are in fact caused by the singularities in the density response function of the electron gas induced by the nesting $[4,5]$. Sodium is a notable exception: although computations show that it does exhibit phonon softening like the other alkalies, its Fermi surface remains exquisitely spherical in the pressure range of interest[2].

Although sodium is not predicted to be a good superconductor, it also presents a quite complex structural phase sequence under pressure. Additionally, the melting curve of sodium presents a pronounced maximum $[6,7]$ which can be explained in terms of a pressure induced decreasing of the Debye temperature associated to a softening of transverse phonon modes [8].

Thus, in order to make the discussion above more tangible, the case of sodium in the fcc structure for pressures between 65 and $105 \mathrm{GPa}$ has been studied. It turns out that the softening observed in computations pertaining to sodium can in fact be understood within the NFE approximation, and that the anomalous features in the phonon spectra are simple Kohn anomalies $[9,10]$, enhanced by pressure.

\section{The nearly free electron model}

The model has been described in great details before and has been found to be very successful in many situations of physical interest[11]. Only a brief summary of the relevant expressions will be presented here. It will be assumed from the outset that the ion-valence electron pseudopotential is local.

It will be convenient to define the unitless form factor as

$$
W(\mathbf{k})=N\left(\epsilon_{F}\right) \frac{v_{p s}(\mathbf{k})}{\epsilon_{e}(\mathbf{k})},
$$

where $N\left(\epsilon_{F}\right)$ is the free electron density of states per unit volume at the free electron Fermi energy, namely

$$
N\left(\epsilon_{F}\right)=\frac{3}{2} \frac{n_{0}}{\epsilon_{F}},
$$

and $\epsilon_{e}$ is the electronic dielectric function. This function can be expressed in terms of the electronic susceptibility of the free 
electron gas,

$$
\chi^{(0)}(\mathbf{k})=-\frac{k_{F}}{\pi^{2} e^{2} a_{0}} F_{L}\left(\frac{|\mathbf{k}|}{2 k_{F}}\right),
$$

and has the form

$$
\epsilon_{e}(\mathbf{k})=1-[1-G(\mathbf{k})] \chi^{(0)}(\mathbf{k}) v_{c}(\mathbf{k}) .
$$

In the above, $k_{F}$ represents the Fermi wave vector, $F_{L}$ is the Lindhard function,

$$
F_{L}(x)=\frac{1}{2}+\frac{1-x^{2}}{4 x} \ln \left|\frac{1+x}{1-x}\right|,
$$

$n_{0}$ is the average number density, $v_{p s}(\mathbf{k})$ is the bare pseudopotential and $v_{c}(\mathbf{k})$ is the Coulomb potential. The local field correction, $G(\mathbf{k})$, accounts for the electron-electron interaction beyond RPA. Of the many forms available in the literature, the approximation of Ishimaru and Utsumi has been retained[12].

It is straightforward to obtain the dynamical matrix within the approximations above[13]:

$$
D_{\alpha \beta}(\mathbf{q})=n_{0} \sum_{\mathbf{k}} \mathbf{k}_{\alpha} \mathbf{k}_{\beta} \phi_{2}(\mathbf{k}) \sum_{\mathbf{K}}\left(\delta_{\mathbf{k}, \mathbf{K}+\mathbf{q}}-\delta_{\mathbf{k}, \mathbf{K}}\right) ;
$$

assuming the bare ion-ion interaction is Coulombic, the effective ionic pair potential is given by[11]

$$
\phi_{2}(\mathbf{k})=v_{c}(\mathbf{k})+\chi(\mathbf{k})\left|v_{p s}(\mathbf{k})\right|^{2} .
$$

The phonon dispersion relation is then given by

$$
M \omega_{v}^{2}(\mathbf{q}) \hat{\mathbf{e}}_{v}(\mathbf{q})=\mathbf{D}(\mathbf{q}) \cdot \hat{\mathbf{e}}_{v}(\mathbf{q}) .
$$

The phonon linewidth can be approximated by[14]

$$
\gamma_{v}(\mathbf{q})=\pi \omega_{v}(\mathbf{q}) \frac{1}{\Omega} \sum_{\mathbf{k}^{\prime}, \mathbf{k} \sigma}\left|g_{v}(\mathbf{q}, \mathbf{k})\right|^{2} \delta\left(\xi_{\mathbf{k}^{\prime}-\mathbf{k}}\right) \delta\left(\xi_{\mathbf{k}^{\prime}}\right),
$$

with

$$
g_{v}(\mathbf{q}, \mathbf{k})=\frac{W(\mathbf{k})}{N\left(\epsilon_{F}\right)} \sqrt{\frac{n_{0} m_{e} \epsilon_{F}}{M \hbar \omega_{v}(\mathbf{q})}} \sum_{\mathbf{K}} \delta_{\mathbf{k}, \mathbf{K}+\mathbf{q}} \frac{\mathbf{k} \cdot \hat{\mathbf{e}}_{v}(\mathbf{q})}{k_{F}} .
$$

For a spherical Fermi surface, which is appropriate in the NFE approximation, it is easy to show that

$$
\frac{1}{\Omega} \sum_{\mathbf{k}^{\prime}} \delta\left(\xi_{\mathbf{k}^{\prime}}\right) \delta\left(\xi_{\mathbf{k}^{\prime}-\mathbf{k}}\right)=\frac{N\left(\epsilon_{F}\right)^{2}}{12 n_{0}} \frac{k_{F}}{k} \theta\left(2 k_{F}-k\right) .
$$

The Eliashberg function can now be expressed as $[14,15,16]$

$$
\alpha^{2} F_{v}(\omega)=\frac{1}{\pi N\left(\epsilon_{F}\right)} \frac{1}{\Omega} \sum_{\mathbf{q}} \frac{\gamma_{v}(\mathbf{q})}{\hbar \omega} \delta\left(\omega-\omega_{v}(\mathbf{q})\right) .
$$

\section{Phonon spectra and Kohn Anomalies}

In order to demonstrate that the features observed in the phonon spectra of sodium are simple Kohn anomalies, the phonons of fcc sodium were computed in the $\Gamma \mathrm{X}$ and $\Gamma \mathrm{K}$ directions at 65,85 and $105 \mathrm{GPa}$ using $a b$ initio methods based on density functional perturbation theory (DFPT)[17] as implemented in the QUANTUM-ESPRESSO [18] package.
The calculations were performed with the choice of the local density approximation (LDA) for the exchange-correlation functional, which was parametrized according to the PerdewZunger description[19]. It should be noted that the use of the generalized gradient approximation (GGA) did not lead to significant differences neither in the band nor the phonon spectra. A Vanderbilt ultrasoft pseudopotential[20], which included $2 s$ and $2 p$ core electrons in the valence, was used throughout this work. Computations were also performed with a pseudopotential that included only the $3 s$ electron in the valence: the results exhibited much stronger Kohn anomalies, but they were discarded as the bands only poorly agreed with those produced using the above mentioned valence 9 pseudopotential in the pressure range of interest. Indeed, the valence 1 pseudopotentials substantially overlapped at the volumes considered, which suggest that their use is inappropriate. This technical detail should be kept in mind when computing phonons at high pressures. Convergence tests suggested the use of a 50 Ry cutoff for the plane-wave basis and 450 for the density, and a $32 \times 32 \times 32$ Monkhorst-Pack mesh[21] for the electronic first Brillouin zone (1BZ) integration. Such a fine mesh was required to converge phonons close to $\Gamma$ point to within $0.03 \mathrm{THz}$. Finally, converging the double delta needed to calculate $a b$ initio the phonon linewidths required a finer $80 \times 80 \times 80$ grid.

The phonons of sodium were then computed using perturbation theory and a weak pseudopotential. This will henceforth be referred to as the linear response method (as opposed to the $a b$ initio method) because the electronic density was computed to linear order in the weak perturbing pseudopotential. An Ashcroft empty-core pseudopotential[22] was used to describe the effective valence electron-ion bare interaction. The pseudopotential is described with a single parameter and has the form

$$
\begin{aligned}
& v_{p s}(\mathbf{r})=\left\{\begin{array}{ll}
0 & r<r_{0} \\
-e^{2} / r & r>r_{0}
\end{array},\right. \\
& v_{p s}(\mathbf{k})=-\frac{4 \pi e^{2}}{k^{2}} \cos \left(k r_{0}\right) .
\end{aligned}
$$

It is then straightforward to compute the phonons using the methods described in the last section.

In order to make contact with the $a b$ initio results, the value of the pseudopotential parameter, $r_{0}$, was chosen such that the longitudinal phonon frequency at $\mathrm{X}$ agreed between the linear response and the $a b$ initio computations, for each value of $r_{s}$ considered. The results of the fit can be seen in Table 1 . The phonon branches at $85 \mathrm{GPa}$ are shown in Figure 1 (results at 65 and $105 \mathrm{GPa}$ were not significantly different), along with the hypothetical dispersion of a zero pressure fcc phase. There, it can be seen that despite the extreme simplicity of the model, the $a b$ initio phonons are qualitatively reproduced by the linear response calculations. In particular, the sound velocities are in good agreement and the phonon softening occurs at sensibly the same wave vectors and the same branches. The results strongly suggest that such a simple approach still captures the basic physics underlying the increasing softening under pressure. 


\begin{tabular}{|c|c|c|}
\hline $\mathrm{P}(\mathrm{GPa})$ & $r_{s}\left(a_{0}\right)$ & $r_{0}\left(a_{0}\right)$ \\
\hline \hline 65 & 2.69 & 1.70 \\
85 & 2.61 & 1.69 \\
105 & 2.54 & 1.67 \\
\hline
\end{tabular}

Table 1: Values of the pseudopotential parameter $r_{0}$ as a function of pressure for a longitudinal phonon fit at $\mathrm{X}$. The pressures are obtained from the $a b$ initio calculated equation of state.

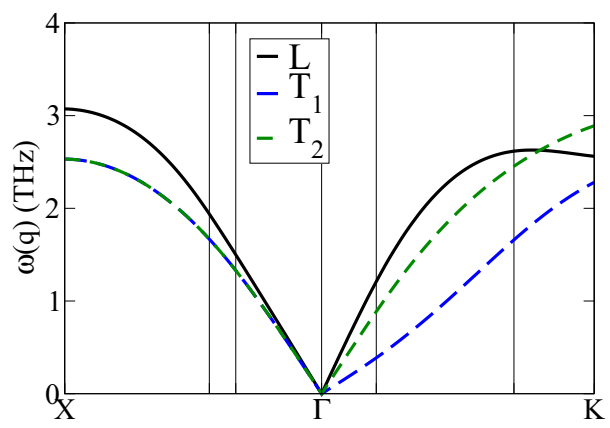

(a) $\mathrm{P}=0 \mathrm{GPa}$

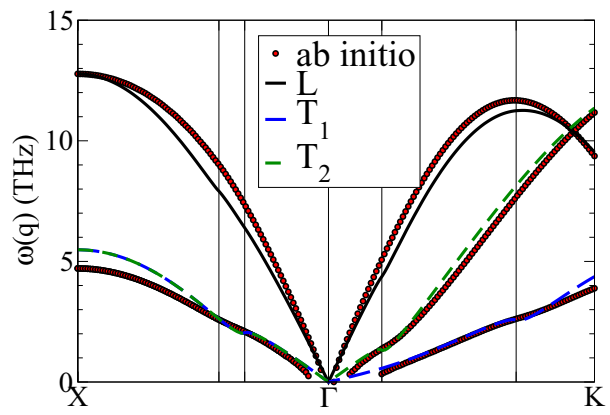

(b) $\mathrm{P}=85 \mathrm{GPa}$

Figure 1: (a) Phonons obtained within linear response for parameters corresponding to zero pressure in an hypothetical fcc phase. No Kohn anomalies appear. (b) Phonons obtained within linear response (full lines) and ab initio (points) for a fit to the longitudinal $\mathrm{X}$ phonon at roughly $85 \mathrm{GPa}$. The longitudinal branch is represented by $\mathrm{L}$, and the transverse branches by $T_{1}$ and $T_{2}$. The vertical lines indicate the positions of the Kohn anomalies (except at $\Gamma$ ). Some $a b$ initio data points are removed near $\Gamma$ because their values could not be properly converged.

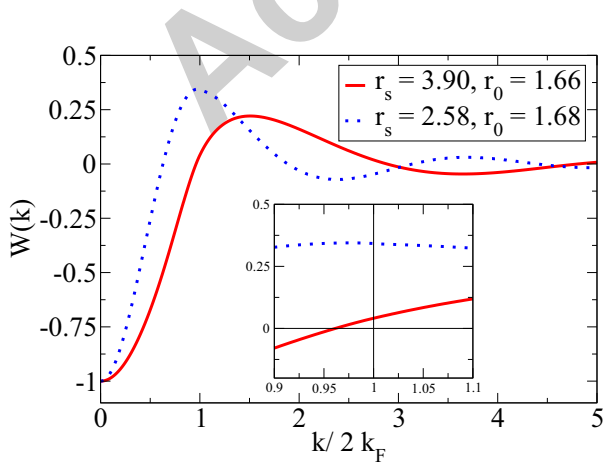

Figure 2: Form factor for $r_{s}=2.58, r_{0}=1.68$, which roughly corresponds to sodium at $90 \mathrm{GPa}$, and $r_{s}=3.9, r_{0}=1.66$, which roughly corresponds to sodium at zero pressure. The form factor is largely enhanced close to $k=2 k_{F}$ at high pressure.



Figure 3: Plot of $\omega_{S}$, as defined in the text, for various values of $r_{0}$. In principle, $r_{0}$ should be state dependent (although Table 1 indicates that it is weakly so in the range of interest), so many values of $r_{0}$ are considered to show that the strength function generically increases as the volume is decreased to the range of interest.

The Kohn anomalies can be analyzed further within the simple model. Since the response function of the electron gas is singular at $2 k_{F}$ (the Lindhard function has infinite slope there), features in the phonon spectrum can be expected whenever[9]

$$
|\mathbf{K}+\mathbf{q}| \simeq 2 k_{F} .
$$

In order to study the amplitude of the anomalies, it is assumed that they are mostly due to the singular behavior of the Lindhard function appearing in $\chi^{(0)}$. The anomalous part of the dynamical matrix in the vicinity of a wave vector which satisfies equation (15) can thus be defined as

$$
\begin{array}{r}
D_{\alpha \beta}^{(a n)}(\mathbf{q})=\frac{N}{\Omega}\left(2 k_{F}\right)^{2} \frac{k_{F}}{2 \pi^{2} e^{2} a_{0}} \frac{\left|v_{p s}\left(2 k_{F}\right)\right|^{2}}{\epsilon_{e}\left(2 k_{F}\right)} \\
\sum_{\mathbf{k}} \hat{\mathbf{k}}_{\alpha} \hat{\mathbf{k}}_{\beta}\left[\sum_{\mathbf{K}_{i}} \delta_{\mathbf{k}, \mathbf{K}_{i}+\mathbf{q}}\right]\left(1-\frac{k}{2 k_{F}}\right) \ln \left|1-\frac{k}{2 k_{F}}\right|,
\end{array}
$$

where the sum on the reciprocal lattice vectors is restricted to the set $\left\{\mathbf{K}_{i}\right\}$ which comply with the Kohn condition for a given $\mathbf{q}$, and it is assumed that it is acceptable to set $k=2 k_{F}$ in all but the singular term. The prefactor is given by

$$
\begin{aligned}
S & =\frac{N}{\Omega}\left(2 k_{F}\right)^{2} \frac{k_{F}}{2 \pi^{2} e^{2} a_{0}} \frac{\left|v_{p s}\left(2 k_{F}\right)\right|^{2}}{\epsilon_{e}\left(2 k_{F}\right)}, \\
& =M \omega_{S}^{2},
\end{aligned}
$$

with

$$
\hbar \omega_{S}=\frac{k_{F} a_{0}}{\pi}\left|\cos \left(2 k_{F} r_{0}\right)\right| \sqrt{\frac{2 m_{e}}{3 M \epsilon_{e}\left(2 k_{F}\right)}} \frac{e^{2}}{a_{0}} .
$$

The function $\omega_{S}$ is plotted in Figure 3. There, it can be seen that it increases dramatically as $r_{s}$ is reduced from its zero pressure value $\left(r_{s} \simeq 4\right)$ to the range of interest. Thus, the prefactor $S$, which is proportional to the square of $\omega_{S}$, must increase by more than an order of magnitude over the same range of $r_{s}$; this dramatic increase in the prefactor of the anomalous dynamical matrix explains why the Kohn anomalies become more prominent under pressure, as can be observed in Figure 1. The analysis can be specialized further to particular branches, and the exact positions of the anomalies can be deduced. 
First, consider the $\Gamma \mathrm{K}$ direction, with the choice

$$
\mathrm{K}=\frac{2 \pi}{a}\left(\frac{3}{4}, \frac{3}{4}, 0\right)
$$

where $a$ is the side length of the unit cell, and define $\mathbf{q}=(\eta+\delta)$ $\mathrm{K}$, where $\eta \mathrm{K}$ satisfies the Kohn condition and $\delta$ is small. The polarizations are given by

$$
\hat{\mathbf{e}}_{L}=\frac{\hat{x}+\hat{y}}{\sqrt{2}}, \quad \hat{\mathbf{e}}_{T_{1}}=\frac{\hat{x}-\hat{y}}{\sqrt{2}}, \quad \text { and } \quad \hat{\mathbf{e}}_{T_{2}}=\hat{z} .
$$

It is straightforward to show that the Kohn condition, given by equation (15), is only satisfied for the following cases:

set 1: $\quad \eta=\frac{4}{3}-\frac{2 \sqrt{2}}{3} \sqrt{\left(\frac{a k_{F}}{\pi}\right)^{2}-1} \simeq 0.2$,

$$
\mathbf{K} \in\left\{\frac{2 \pi}{a}(-1,-1,1), \frac{2 \pi}{a}(-1,-1,-1)\right\},
$$

set 2: $\quad \eta=\frac{4}{3}-\frac{4}{3} \sqrt{\frac{1}{2}\left(\frac{a k_{F}}{\pi}\right)^{2}-1} \simeq 0.7$,

$$
\mathbf{K} \in\left\{\frac{2 \pi}{a}(-2,0,0), \frac{2 \pi}{a}(0,-2,0)\right\} .
$$

In the vicinity of $\eta \simeq 0.2$, the anomalous dynamical matrix is given by

$$
\begin{gathered}
\mathbf{D}^{(a n)}(\delta)=\frac{3 \alpha M \omega_{S}^{2}}{\left(2 \alpha^{2}+1\right)^{2}}\left(\begin{array}{ccc}
\alpha^{2} & \alpha^{2} & 0 \\
\alpha^{2} & \alpha^{2} & 0 \\
0 & 0 & 1
\end{array}\right) \delta \ln |\delta|, \\
\alpha=\left(1-\frac{3}{4} \eta\right) \simeq 0.85 .
\end{gathered}
$$

In this last expression only the term proportional to $\delta \ln |\delta|$ has been retained, and $\alpha$ has been defined in order to make the equation more legible. Equation (24) implies

$$
\mathbf{D}^{(a n)} \hat{\mathbf{e}}_{T_{1}}=\mathbf{0}
$$

and there thus can be no anomaly in the $T_{1}$ branch. Furthermore,

$$
\hat{\mathbf{e}}_{L} \cdot \mathbf{D}^{(a n)}(\delta) \cdot \hat{\mathbf{e}}_{L}=2 \alpha^{2} \hat{\mathbf{e}}_{T_{2}} \cdot \mathbf{D}^{(a n)}(\delta) \cdot \hat{\mathbf{e}}_{T_{2}} ;
$$

since $2 \alpha^{2} \simeq 1.5$, the longitudinal anomalous contribution to the dynamical matrix is of similar magnitude in the $T_{1}$ and $L$ branches. A similar analysis can be performed for other points where the Kohn condition is satisfied. Results are summarized in Table 2.

On the face of it, however, it is surprising that the longitudinal branches do not exhibit strongly anomalous behavior. Consider a phonon branch near a singularity with polarization $\hat{\mathbf{e}}$; then,

$$
\begin{aligned}
M \omega(\delta)^{2} & =\hat{\mathbf{e}} \cdot \mathbf{D}(\delta) \cdot \hat{\mathbf{e}} \\
& \simeq M \omega_{R}(\delta)^{2}+\Lambda M \omega_{S}^{2} \delta \ln |\delta|,
\end{aligned}
$$

where $\omega_{R}(\delta)$ is the non-anomalous part of the spectrum and the last term is equal to $\hat{\mathbf{e}} \cdot \mathbf{D}^{(a n)}(\delta) \cdot \hat{\mathbf{e}}$. The phonon frequency can be expanded as

$$
\omega(\delta) \simeq \sqrt{\omega_{R}^{2}+2 \omega_{R} \omega_{R}^{\prime} \delta+\omega_{S}^{2} \Lambda \delta \ln |\delta|},
$$

\begin{tabular}{|c|c|c|c|c|}
\hline Direction & set & $\eta$ (approx.) & polarization & $\Lambda$ (approx.) \\
\hline$\Gamma \mathrm{K}$ & 1 & 0.2 & $T_{2}$ & 0.4 \\
& & & $L$ & 0.6 \\
\hline & 2 & 0.7 & $T_{1}$ & 0.5 \\
& & & $L$ & 0.1 \\
\hline \hline$\Gamma \mathrm{X}$ & 1 & 0.3 & $T_{1}, T_{2}$ & 0.2 \\
& & & $L$ & 0.5 \\
\hline & 2 & 0.4 & $L$ & 0.6 \\
\hline
\end{tabular}

Table 2: Summary of the various results describing the strength of the Kohn anomalies along $\Gamma \mathrm{X}$ and $\Gamma \mathrm{K} . \eta$ is defined such that $\mathbf{q}=\eta \mathrm{K}$ or $\mathbf{q}=\eta \mathrm{X} . \Lambda$ is defined in equation (28). Only the branches with Kohn anomalies are mentioned.

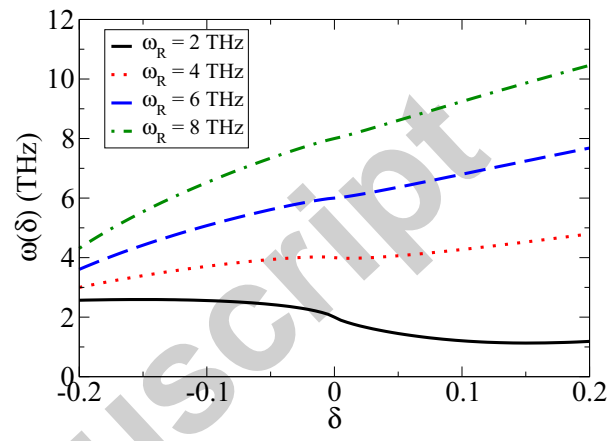

Figure 4: Frequency in the vicinity of a Kohn anomaly, in the approximations of equation (32), for various reasonable values of $\omega_{R}$ and $\omega_{S}=6 \mathrm{THz}$. The graph makes it clear that the "kink" is quickly ironed away as $\omega_{R}$ increases, for equal $\omega_{S}$

where $\omega_{R} \equiv \omega_{R}(\delta=0)$ and

$$
\omega_{R}^{\prime}=\left.\frac{d}{d \delta} \omega_{R}(\delta)\right|_{\delta=0} .
$$

In the above, $\Lambda$ is a numerical factor which depends on the branch considered (the values of $\Lambda$ for each anomaly considered are given in Table 2). For the sake of simplicity, let's consider a Debye model for the regular part of the spectrum. Then, $\omega_{R}(\mathbf{q})=c|\mathbf{q}|$, and $\omega_{R}^{\prime} \simeq \omega_{D} \simeq 2 \omega_{R}$, where $\omega_{D}$ is the Debye frequency, and it is assumed that the anomaly occurs roughly in mid-branch (i.e. $\eta \simeq 0.5$ ). Also, according to Table $2, \Lambda$ is at most roughly equal to 0.5 . Then, in the vicinity of an anomaly in a longitudinal branch,

$$
\omega(\delta) \simeq \sqrt{\omega_{R}^{2}+4 \omega_{R}^{2} \delta+\frac{1}{2} \omega_{S}^{2} \delta \ln |\delta|} .
$$

This function is plotted in Figure 4 in the vicinity of $\delta=0$ for the choice $\omega_{S} \simeq 6 \mathrm{THz}$ (roughly the largest value attained in the model, in the range of interest; see Figure 3) for various values of $\omega_{R}$. The graph makes it clear that, for everything else being equal, the "kink" due to the Kohn anomaly will appear much weaker in a high branch (typically longitudinal) than in a low branch (transverse).

\section{Linewidths and electron-phonon coupling}

The linewidths were computed in the NFE model using the values of the parameters $r_{0}=1.68$ and $r_{s}=2.58$, which according to Table 1 roughly correspond to sodium at $90 \mathrm{GPa}$. The 
results can be seen in Figure 5. The main features displayed by the linewidths are that large discontinuous contributions for the transverse branches appear where the Kohn condition is satisfied. These features are trivially explained by the definition of the linewidths, equation (9). Indeed, the linewidths have the form

$$
\gamma_{v}(\mathbf{q}) \sim \sum_{\mathbf{K}} \theta\left(2 k_{F}-|\mathbf{K}+\mathbf{q}|\right)\left((\mathbf{K}+\mathbf{q}) \cdot \hat{\mathbf{e}}_{v}(\mathbf{q})\right)^{2} ;
$$

thus there can only be non-longitudinal coupling for Umklapp processes (namely, $\mathbf{K} \neq \mathbf{0}$ : in high symmetry directions such as $\Gamma \mathrm{X}$ or $\Gamma \mathrm{K}$, the polarization of the longitudinal branch truly is longitudinal). Furthermore, it is clear that the onset of the contribution of the Umklapp processes will coincide with the Kohn condition. It is clear from Figure 5 that this also holds true for the $a b$ initio results. Finally, as can be seen in Figure 2, the form factor is largely enhanced at higher pressure, leading to substantial Umklapp contribution to the linewidths.

As can be seen in Figure 6, the large Umklapp transverse contributions to the linewidths are reflected in an Eliashberg function which is largest at low frequencies. This leads to a fairly large electron-phonon coupling $(\lambda \simeq 0.5)$ with a dominating contribution coming from the low frequency region of the spectrum. This value is in rough agreement with that obtained by an $a b$ initio calculation under similar conditions[23] (it is noteworthy that the $a b$ initio computations presented here do not reproduce the imaginary transverse phonon frequencies along the (110) direction mentioned by Christensen and Novikov). A simple calculation using the McMillan equation[24] with the standard $\mu^{*}=0.1$ yielded $T_{c} \simeq 2.5 \mathrm{~K}$, with $\omega_{\text {log }} \simeq 167 \mathrm{~K}$, again in rough agreement with published results[23].

The global picture is now very clear. Within the model described above, pressure (namely, the value of $r_{s}$ ) can be tuned such that the maximum of the form factor occurs close to $2 k_{F}$. This leads to strong Kohn anomalies in the transverse phonon spectrum and large contributions to the Umklapp transverse linewidths at exactly the same positions in the 1BZ. This leads to a transverse mode dominated Eliashberg function and a large enhancement of the electron-phonon coupling because the phonon frequencies are reduced around the Kohn anomalies and the linewidths are increased.

\section{Conclusion}

The work presented here suggests that the anomalous phonon spectrum of sodium in the 65-105 GPa range can be understood in terms of a NFE model. The "kinks" in the phonon branches correspond to Kohn anomalies and their dramatic increase in amplitude with pressure in the transverse branches is due to the sharp increase in the prefactor of the anomalous dynamical matrix, which in turn is caused by the interplay of the two important length scales in the problem, namely $r_{s}$ (which appears in $k_{F}$ ), and $r_{0}$, which describes roughly the size of the sodium ion.

The increase of the form factor under pressure also leads to a sharp increase of the linewidths right where the Kohn condition is satisfied because of Umklapp contributions. In conjunction

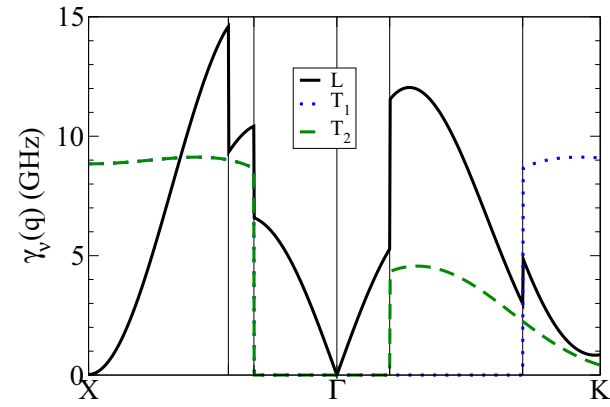

(a) Linear response

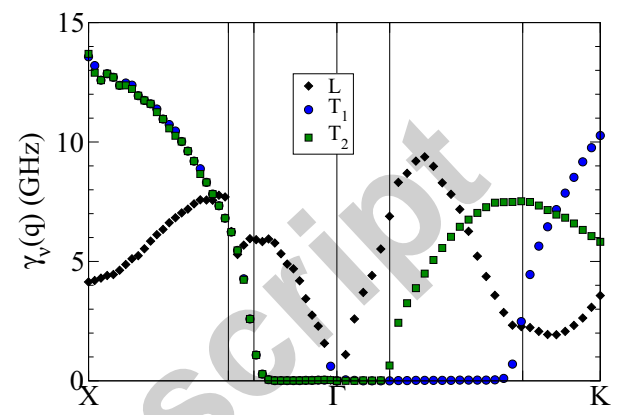

(b) Ab initio

Figure 5: (a) Linewidths in the NFE approximation and (b) computed ab initio for sodium in the fcc lattice, along the special directions $\Gamma X$ and $\Gamma K$. The parameters are $r_{0}=1.68$ and $r_{s}=2.58$, which correspond roughly to a pressure of $90 \mathrm{GPa}$. The vertical lines away from $\Gamma$ indicate the positions where the Kohn condition is satisfied.

with the softening of the phonon modes, this leads to an Eliashberg function which is dominated by the transverse frequency contributions and an unexpectedly large electron-phonon coupling.

Although the model used was very simple, it is expected that the trends observed should be quite general. Pressure can be used to maximize the pseudopotential form factor where the response function of the electron gas has a very large derivative (roughly $2 k_{F}$ for a roughly spherical Fermi surface); this should lead to both a softening of the transverse phonon modes (Kohn anomalies) and large Umklapp contributions to the linewidths, thus enhancing superconductivity.

This work was partially supported by the National Science Foundation (Grant DMR-0601461). B. R. gratefully acknowledges the Cornell Center for Materials Research for the allocation of computational resources. B. R. is also grateful for fruitful discussion with Professor N.W. Ashcroft at Cornell University. The research herein presented was partially performed under project BFM2003-04428, funded by the Spanish Ministry of Education and Science. I.E would like to thank the Basque Department of Education, Universities and Research for financial help. The authors are also thankful to SGI-IZO SGIker UPV-EHU for the allocation of computational resources. 


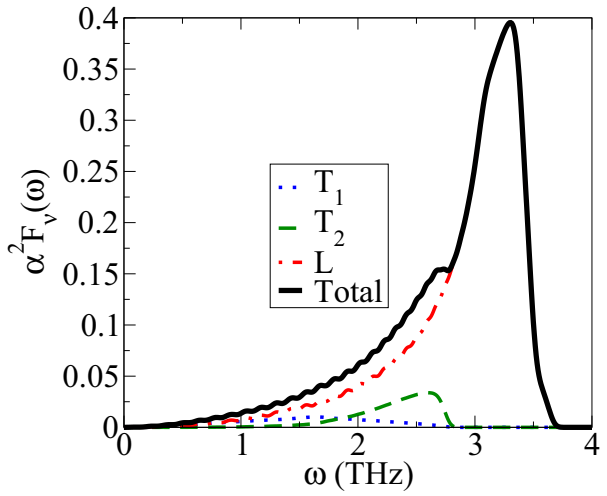

(a) $\mathrm{P}=0 \mathrm{GPa}$

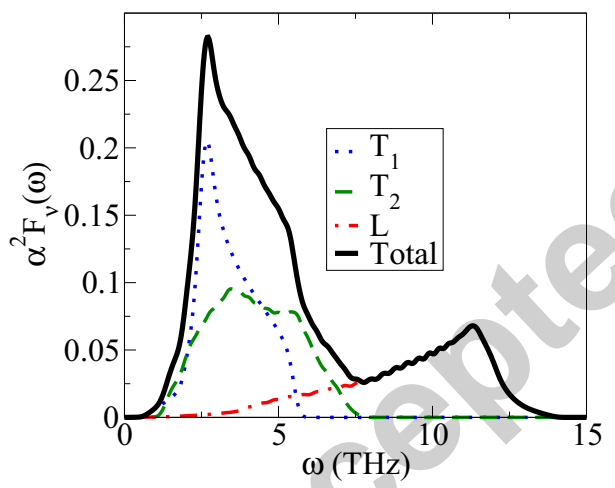

(b) $\mathrm{P}=90 \mathrm{GPa}$

Figure 6: Eliashberg function computed in the NFE model for (a) an hypothetical fcc phase of sodium at zero pressure $\left(r_{0}=1.66\right.$ and $\left.r_{s}=3.9\right)$, and (b) at a pressure of about $90 \mathrm{GPa}\left(r_{0}=1.68\right.$ and $\left.r_{s}=2.58\right)$. The transverse contribution increases dramatically with pressure.

\section{References}

[1] M. L. Cohen, Electron-phonon-induced superconductivity, Journal of Superconductivity and Novel Magnetism 19 (3-5) (2006) 283-290.

[2] Y. Xie, J. S. Tse, T. Cui, A. R. Oganov, Z. He, Y. Ma, G. Zou, Electronic and phonon instabilities in face-centered-cubic alkali metals under pressure studied using ab initio calculations, Physical Review B 75 (6) (2007) 064102.

[3] A. Rodriguez-Prieto, A. Bergara, V. M. Silkin, P. M. Echenique, Complexity and fermi surface deformation in compressed lithium, Physical Review B 74 (17) (2006) 172104

[4] A. Rodriguez-Prieto, V. M. Silkin, A. Bergara, Nesting induced peierlstype instability for compressed li-ci 16, J. Phys. Proc. Jpn 76 (9) (2007) 21-2.

[5] A. Rodriguez-Prieto, M. Martinez-Canales, V. M. Silkin, A. Bergara, Anomalous static electronic screening in compressed lithium, Journal of Physics: Conference Series 121 (1) (2008) 012007.

[6] E. Gregoryanz, O. Degtyareva, M. Somayazulu, R. J. Hemley, H. kwang Mao, Melting of dense sodium, Physical Review Letters 94 (18) (2005) 185502.

[7] J.-Y. Raty, E. Schwegler, S. A. Bonev, Electronic and structural transitions in dense liquid sodium, Nature 449 (2007) 448-451, 10.1038/nature06123.

[8] M. Martinez-Canales, A. Bergara, Lindemann criterion and the anomalous melting curve of sodium, Journal of Physics and Chemistry of Solids 69 (9) (2008) $2151-2154$.

[9] W. Kohn, Image of the fermi surface in the vibration spectrum of a metal, Phys. Rev. Lett. 2 (9) (1959) 393-394.

[10] E. J. Woll, W. Kohn, Images of the fermi surface in phonon spectra of metals, Phys. Rev. 126 (5) (1962) 1693-1697.

[11] J. Hafner, From Hamiltonians to Phase Diagrams, Springer-Verlag, 1987

[12] S. Ichimaru, K. Utsumi, Analytic expression for the dielectric screening function of strongly coupled electron liquids at metallic and lower densities, Phys. Rev. B 24 (12) (1981) 7385-7388.

[13] N. W. Ashcroft, D. Mermin, Solid State Physics, Thompson Learning, Inc., 1976.

[14] P. B. Allen, Neutron spectroscopy of superconductors, Phys. Rev. B 6 (7) (1972) 2577-2579

[15] P. B. Allen, B. Mitrovic, Theory of superconducting $\mathrm{T}_{c}$, Solid State Physics 37 (1982) 1.

[16] J. P. Carbotte, R. C. Dynes, Superconductivity in simple metals, Phys. Rev. 172 (2) (1968) 476-484

[17] S. Baroni, S. de Gironcoli, A. Dal Corso, P. Giannozzi, Phonons and related crystal properties from density-functional perturbation theory, Rev. Mod. Phys. 73 (2) (2001) 515-562.

[18] P. Gianozzi et al., http://www.quantum-espresso.org/.

[19] J. P. Perdew, A. Zunger, Self-interaction correction to density-functional approximations for many-electron systems, Phys. Rev. B 23 (10) (1981) 5048-5079.

[20] D. Vanderbilt, Soft self-consistent pseudopotentials in a generalized eigenvalue formalism, Phys. Rev. B 41 (11) (1990) 7892-7895.

[21] H. J. Monkhorst, J. D. Pack, Special points for brillouin-zone integrations, Phys. Rev. B 13 (12) (1976) 5188-5192.

[22] N. W. Ashcroft, Electron-ion pseudopotentials in metals, Phys. Lett. 23 (1966) 48-53.

[23] N. E. Christensen, D. L. Novikov, Calculated superconductive properties of $\mathrm{Li}$ and Na under pressure, Physical Review B 73 (22) (2006) 224508.

[24] W. L. McMillan, Transition temperature of strong-coupled superconductors, Phys. Rev. 167 (2) (1968) 331-344. 